\title{
HIDRÓLISE ENZIMÁTICA DE PAPEL DE ESCRITÓRIO DESCARTADO COM E SEM PRÉ-TRATAMENTO COM ÁCIDO SULFÚRICO DILUÍDO
}

\author{
J. M. T. S. ROCHA, B. R. A. ALENCAR, H. G. MOTA e E. R. GOUVEIA \\ Universidade Federal de Pernambuco, Departamento de Antibióticos \\ E-mail para contato: estergouveia@gmail.com
}

\begin{abstract}
RESUMO - O objetivo deste trabalho foi realizar hidrólises enzimáticas de papel de escritório descartado com e sem pré-tratamento com ácido sulfúrico diluído, visando a produção de bioetanol. Foram realizadas hidrólises com 2, 4, $810 \% \mathrm{~m} / \mathrm{V}$ de sólido. A quantidade de enzima utilizada foi a mesma independente do aumento da massa de papel. As concentrações de glicose e xilose foram quantificadas por cromatografia líquida de alta eficiência. Houve aumento das concentrações destes carboidratos à medida que aumentou a massa de papel até $8 \% \mathrm{~m} / \mathrm{V}$. Não foi possível retirar amostra nas hidrólise com 8 ou 10 $\% \mathrm{~m} / \mathrm{V}$ e sem pré-tratamento, uma vez que não ocorreu a liquefação do papel. Maiores concentrações carboidratos foram obtidas na hidrólise com pré-tratamento e $8 \% \mathrm{~m} / \mathrm{V}$, apesar de o rendimento ter sido menor do que quando foi utilizado $2 \% \mathrm{~m} / \mathrm{V}$, provavelmente devido à quantidade de enzima ter sido a mesma para ambos os casos.
\end{abstract}

\section{INTRODUÇÃO}

A tecnologia para a produção de etanol a partir de material lignocelulósico é baseada principalmente no pré-tratamento, hidrólise química ou enzimática, separação do produto de fermentação e destilação (Dubey et al., 2012). O papel é um material lignocelulósico, cuja composição química varia de acordo com a madeira da qual foi originado. Material lignocelulósico como papel de escritório contem $56 \%, 14 \%$ e $6 \%$ de celulose, hemicelulose e lignina, respectivamente (Wang et al., 2012).

A hidrólise enzimática da celulose é realizada por um conjunto de enzimas celulolíticas, composto de uma mistura de endo- $\beta$-1,4-glucanases, exo- $\beta$-1,4-glucanases e $\beta$-glucosidase (Palmqvist \& Hahn-Hagerdal 2000). Estas enzimas atuam em diferentes sítios da cadeia celulósica de forma sinérgica: as exo-glucanases, também conhecidas como celobiohidrolases, atuam clivando ligações glicosídicas nas extremidades da cadeia, obtendo-se as unidades de celobiose; as endo-glucanases clivam regiões amorfas internas da cadeia celulósica e as $\beta$ - 
glucosidases clivam a celobiose em unidades de glicose (Galbe \& Zacchi, 2002).

O objetivo deste trabalho foi realizar hidrólises enzimáticas com diferentes massas de papel de escritório descartado, sem e com pré-tratamento com ácido sulfúrico diluído, visando a produção de bioetanol.

\section{MATERIAIS E MÉTODOS}

\subsection{Material Lignocelulósico}

Foi utilizado papel de escritório descartado do Departamento de Antibióticos da UFPE.

\subsection{Hidrólise Enzimática do Papel de Escritório Descartado}

As hidrólises, com 2, 4, 8 e $10 \% \mathrm{~m} / \mathrm{v}$ de papel, foram realizadas em frascos de Erlenmeyers, com tampão citrato de sódio ( $\mathrm{pH}$ igual a 4,8). Preparações comerciais de celulases (Celluclast $1.5 \mathrm{~L}-$ $2 \mathrm{~mL} ; 119 \mathrm{FPU} / \mathrm{mL})$ e $\beta$-glucosidase $(1 \mathrm{~mL})$, ambas da Novozyme foram utilizadas. A atividade enzimática da Celluclast $1.5 \mathrm{~L}$ foi determinada com unidade de papel de filtro por $\mathrm{mL}$ segundo o método de Ghose (1987). Os frascos, em todas as hidrólises enzimáticas, foram mantidos em mesa incubadora rotativa, a $50^{\circ} \mathrm{C}$ e $150 \mathrm{rpm}$. Após a filtração das amostras em membrana de $0,45 \mu \mathrm{m}$, os filtrados foram utilizados para a quantificação de glicose e xilose por cromatografia líquida de alta eficiência.

Nas hidrólises com pré-tratamento, o material foi inicialmente colocado em frascos de Erlenmeyer, onde foram adicionados $100 \mathrm{~mL}$ de $\mathrm{H}_{2} \mathrm{SO}_{4}$ a $1 \% \mathrm{~V} / \mathrm{V}$. O frasco foi acondicionado em mesa incubadora rotativa, a temperatura de $50^{\circ} \mathrm{C}$ e rotação de $150 \mathrm{rpm}$, durante 3 horas. Após este período, o material foi centrifugado por 10 minutos, a $10000 \mathrm{rpm}$ e o decantado foi utilizado nas hidrólises enzimáticas. 


\subsection{Quantificação de Carboidratos}

As amostras, retiradas ao final de cada hidrólise enzimática, foram analisadas por meio da técnica de Cromatografia Líquida de Alta Eficiência (CLAE), utilizando uma coluna Aminex HPX$87 \mathrm{H}^{+}$(300 mm x 7,8 mm, Bio-Rad Laboratories, Richmond, CA, USA), a $60^{\circ} \mathrm{C}$ e detecção de índice de refração. Foram realizadas injeções de $5 \mu \mathrm{L}$, com vazão de $0,6 \mathrm{~mL} / \mathrm{min}$, utilizando como fase móvel $\mathrm{H}_{2} \mathrm{SO}_{4}$ a $5 \mathrm{mM}$. As concentrações de açúcares redutores foram determinadas pelo método do ácido dinitrosalicílico (Miller, 1959).

\subsection{Rendimento}

O rendimento das hidrólises com relação à formação de glicose e xilose foi determinado pela equação (1).

$Y(\%) \square \frac{\text { ART..V }}{\mathrm{m}} .100$

ART: concentração de açúcares redutores (em g/L);

$\mathrm{V}$ : volume da mistura reacional (em L);

m: massa de papel (em g).

\section{RESULTADOS}

As concentrações de glicose e de xilose aumentaram com o acréscimo da massa de papel (Figura 1), nas hidrólises sem pré-tratamento, quando foram utilizados $2 \mathrm{ou} 4 \mathrm{~g}$ de papel. O maior aumento foi obtido na concentração de glicose. Nas hidrólises com 8 ou $10 \% \mathrm{~m} / \mathrm{V}$ e sem prétratamento, não ocorreu a liquefação do papel, o que impossibilitou a retirada de amostra em todo o período avaliado. 


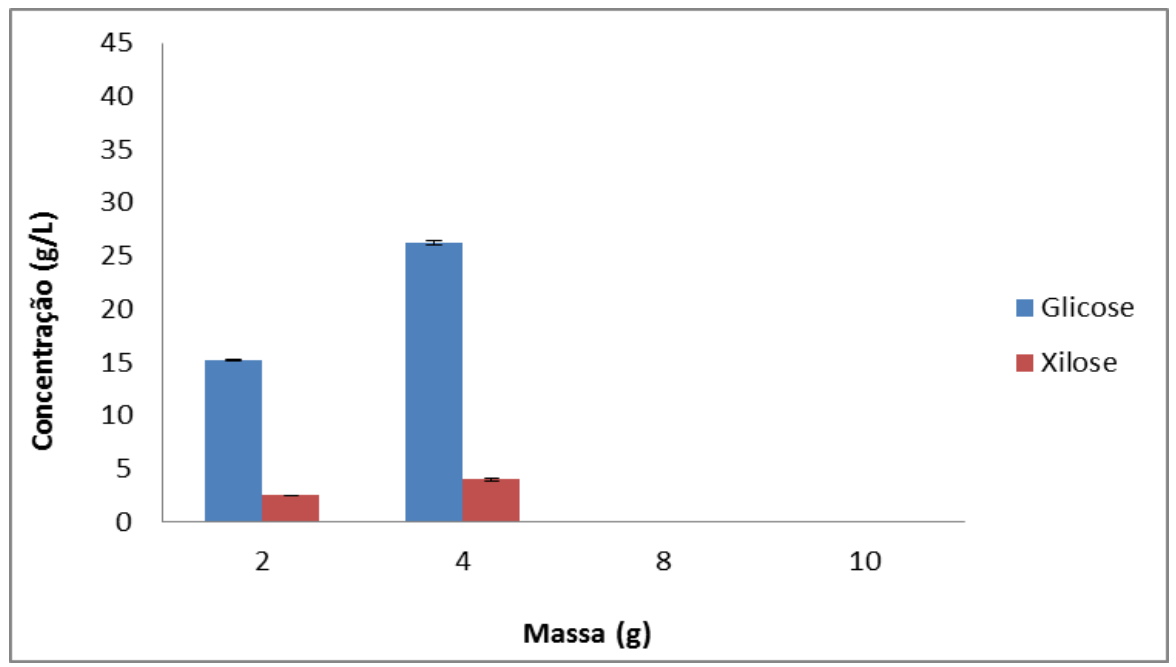

Figura 1. Concentração de glicose e de xilose após 96 horas de hidrólise e sem prétratamento.

As maiores concentrações de glicose e xilose foram obtidas na hidrólise com prétratamento e utilizando $8 \% \mathrm{~m} / \mathrm{V}$. Tanto em $2 \% \mathrm{~m} / \mathrm{V}$, quanto em $4 \% \mathrm{~m} / \mathrm{V}$ houve uma redução nas concentrações dos carboidratos, nas hidrólises com pré-tratamento (Figura 2) em relação aos resultados sem pré-tratamento (Figura 1).

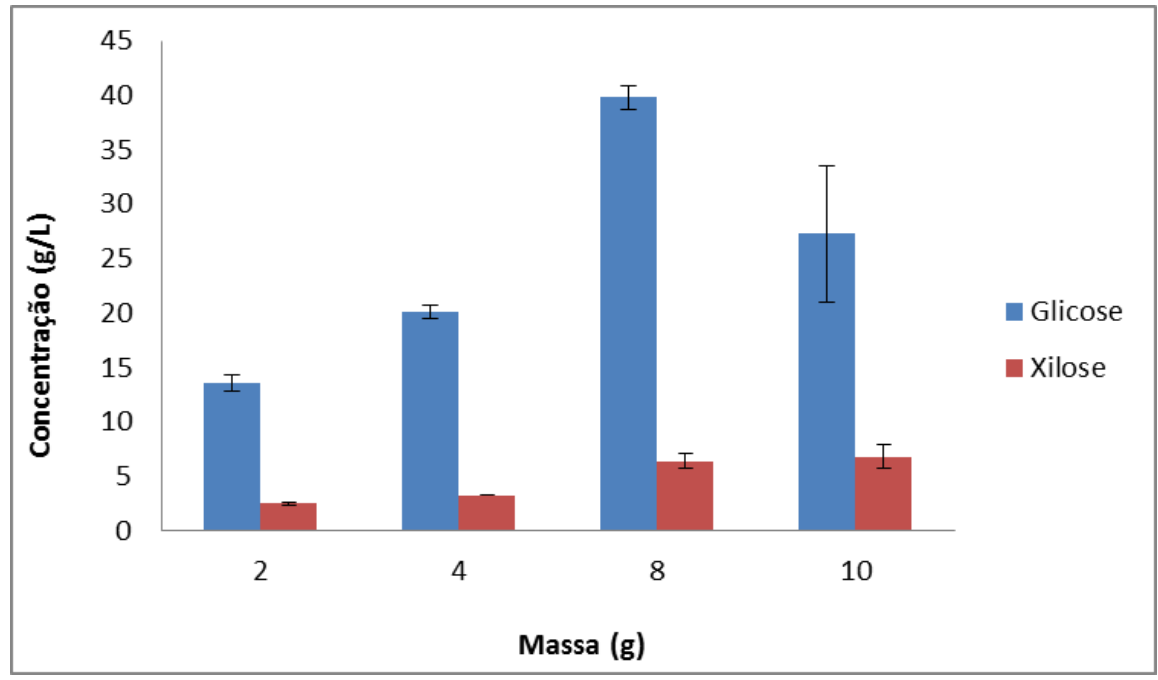

Figura 2. Concentração de glicose e de xilose após 96 horas de hidrólise e com pré-tratamento. 
Apesar do aumento da carga de sólidos de 8 para $10 \% \mathrm{~m} / \mathrm{V}$, houve menor formação de glicose quando foi utilizado $10 \% \mathrm{~m} / \mathrm{V}$. Isso ocorreu provavelmente devido à carga enzimática ter sido a mesma em todas as hidrólises, independente do aumento do substrato (Figura 2).

Os rendimentos são apresentados na Figura 3. Observa-se que houve maior rendimento com $2 \% \mathrm{~m} / \mathrm{V}$, quando não foi utilizado pré-tratamento. 0 rendimento com $4 \% \mathrm{~m} / \mathrm{V}$ também sem pré-tratamento foi menor do que com $2 \% \mathrm{~m} / \mathrm{V}$, o que possivelmente ocorreu por ter sido utilizada a mesma quantidade de enzimas para uma maior massa de papel. Por outro lado, o rendimento com o pré-tratamento e utilizando $8 \mathrm{~g}$ de papel foi semelhante ao rendimento obtido por $4 \mathrm{~g}$ com pré-tratamento. 0 menor rendimento foi obtido com $10 \% \mathrm{~m} / \mathrm{V}$.

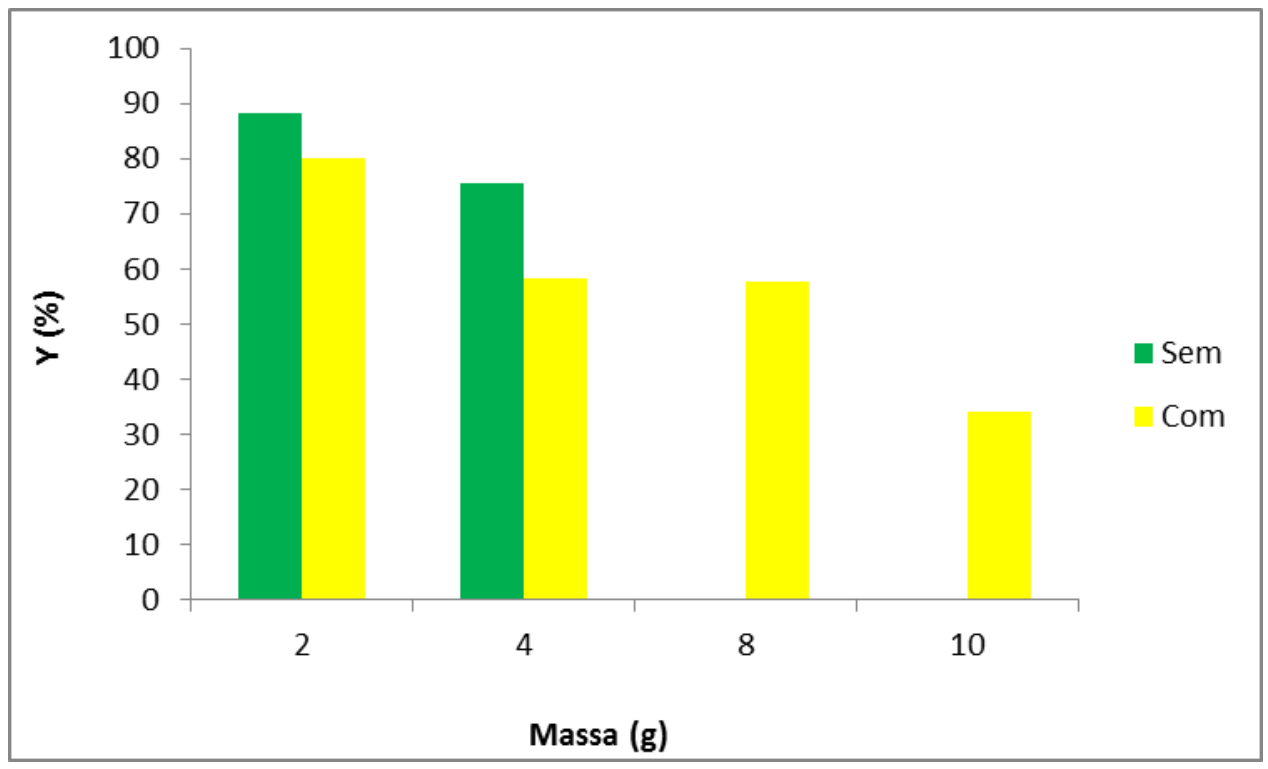

Figura 3 - Rendimento das hidrólises enzimáticas, com 96 horas, sem e com prétratamento.

No estudo da hidrólise enzimática de papel de escritório descartado, quatro fatores foram avaliados: o tempo de hidrólise, a carga enzimática, a adição de surfactante e o pré-tratamento com ácido fosfórico (Chu e Feng, 2013). O rendimento máximo de açúcar foi $82 \%$ para $50 \mathrm{mg}$ de papel, com 20 horas de hidrólise, com pré-tratamento com ácido fosfórico, mas sem adicionar surfactante. Por outro lado, a hidrólise enzimática de um papel offset, semelhante ao papel utilizado neste trabalho, foi estudada por Brummer et al. (2013), os quais obtiveram rendimento de apenas 18,8 \%. Isso pode ter ocorrido devido ao pré-tratamento ter sido realizado apenas por moagem mecânica e lavagem com água destilada. 
O pré-tratamento com ácido sulfúrico diluído foi fundamental para as hidrólises enzimáticas com alta carga de sólidos (8 ou $10 \% \mathrm{~m} / \mathrm{V})$. Na produção de bioetanol, a concentração inicial de açúcares fermentescíveis é um fator preponderante para a obtenção de maior concentração do produto. Daí a seleção de $8 \% \mathrm{~m} / \mathrm{V}$ para a carga de enzimas utilizada neste trabalho, uma vez que com esta condição foi obtida a maior concentração de glicose e xilose.

\section{REFERENNCIAS}

BRUMMER, V.; JURENA, T.; HLAVACEK, V.; Omelkova, J.; Bebar, L.; Gabriel, P.; Stehlik, P.; Enzymatic hydrolysis of pretreated waste paper - Source of raw material for production of liquid biofuels. Bioresource Technology, v. 152, p. 543-54, 2014.

CHU, K. H.; FENG, X. Enzymatic conversion of newspaper and office paper to fermentable sugars. Process Safety and Environmental Protection, v. 9, p. 123-130, 2013.

DUBEY, A. K.; GUPTAB, P. K.; GARGA, N.; NAITHANIB, S. Bioethanol production from waste paper acid pretreated hydrolyzate with xylose fermenting Pichia stipitis. Carbohydrate Polymers, v. 88 , p. 825-829, 2012.

GALBE, M. \& ZACCHI, G. A review of the prodution of ethanol from softwood. Applied Microbiology and Biotechnology, v. 59, p. 618-628, 2002.

GHOSE, T. K. Measurement of cellulase activities. Pure Appl Chem, v. 68, p. 59:257-68, 1987.

MILLER, G. L. Use of dinitrosalicylic acid reagent for determination of reducing sugar. Anal. Chem., v. 31, p. 420-428, 1959.

PALMQVIST, E. \& HAHN-HAGERDAL, B. Fermentation of lignocellulosic hydrolysates. II: inhibitors and mechanisms of inhibition. Bioresource Technology, v. 74, p. 25-33, 2000.

WANG, L.; SHARIFZADEH, M.; TEMPLER, R.; MURPHY, J. Bioethanol production from various waste papers: Economic feasibility and sensitivity analysis. Applied Energy, v. 111, p. 1172-1182, 2012. 